Revista Colombiana de Obstetricia y Ginecología Vol. 56 No.2 • 2005 • (176-179)

Reporte de CAso

\title{
RABDOMIOMA: TUMOR DEL CORAZÓN FETAL. DIAGNÓSTICO PRENATAL
}

\author{
Prenatal diagnosis of tumour of the foetal heart (rhabdomyoma) \\ Juan Carlos Otero, M.D.* \\ Recibido: abril 11/05 - Revisado: mayo 25/05 - Aceptado: junio 8/05
}

\section{RESUMEN}

Los tumores primarios del corazón son raros. En estudios de autopsias la incidencia puede llegar a ser hasta del 0,002-0,250\%; el rabdomioma es el tumor benigno más común (50-78\%), y se asocia con esclerosis tuberosa en $72 \%$ de los casos. ${ }^{1}$ En la gran mayoría, este tumor tiende a regresar.

Los síntomas típicos son secundarios a los efectos ocasionados por las alteraciones de la geometría ventricular izquierda (gasto cardíaco y fracción de eyección). El hydrops y las arritmias son, en estos casos, las principales causantes de la muerte repentina.

La conducción atrioventricular anormal se debe a la interrupción por parte del tumor, del tejido fino nodal o septal. Los niños afectados tienen una edad de gestación promedio entre 22 a 36 semanas, ${ }^{2}$ y su pronóstico depende en especial de la presencia de arritmias y de esclerosis tuberosa. ${ }^{3}$ Como tratamiento prenatal se ha intentado el uso de la digital y otros medicamentos.

Previa autorización de la paciente, se presenta este caso para ilustrar acerca de las posibilidades de diagnóstico y tratamiento antenatal de esta patología, de rara presentación en la práctica.

Palabras clave: rabdomioma, esclerosis tuberosa, arritmias.

* Ginecólogo-Obstetra. Especialista en Medicina Materno Fetal (Universidad de Costa Rica). Fellow en Ultrasonido Obstétrico, Hospital Karolinska (Suecia). Ecocardiografía Fetal (Texas Tech University, Estados Unidos). Perinatólogo (Clínica Materno Infantil San Luis). Profesor de cátedra de alto riesgo obstétrico (Hospital Universitario de Santander)

Correo electrónico: juan_carlos_otero@yahoo.com

\section{SUMMARY}

Primary heart tumours are rare. Overall incidence ranges from $0.002-0.250 \%$ in autopsy. Rhabdomyoma is the most common benign tumour (50-78\%); it has $72 \%$ associated with tuberous sclerosis. This tumour tends to return in most cases.

Typical symptoms are secondary to adverse effects caused by left ventricular geometry (cardiac wear and ejection). Hydrops foetalis and arrhythmia often lead to sudden death.

Abnormal atrioventricular (AV) conduction is due to a tumour disrupting nodal or septal conduction tissue. Gestational age has ranged from 22 to 36 weeks when diagnosis has been made. Prognosis is frequently associated with arrhythmia and tuberous sclerosis. Prenatal digitalis therapy and other medications have been tried in cases of foetuses suffering from heart failure or arrhythmia.

The patient's prior consent has been given here to report a case of ante-natal diagnosis of cardiac rhabdomyoma to teach the diagnosis and management of foetuses suffering from this rare pathology.

Keywords: rhabdomyoma, tuberous sclerosis, arrhythmia. 


\section{CASO CLÍNICO}

Mujer de 39 años de edad, referida por ruptura prematura de membranas con 33,1 semanas de gestación por fecha de última regla y antecedente de dos cesáreas anteriores. La paciente es ingresada para estudio de la ruptura de membranas.

Dentro de las pruebas de rutina se solicitó un ultrasonido obstétrico encontrándose los siguientes parámetros:

* Índice de líquido amniótico en 7,6.

* Crecimiento dentro del percentil 50 para la edad.

* Masa hiperecogénica en la imagen de cuatro cámaras.

Por lo anterior, se consideró realizar un ecocardiografía fetal que reportó entonces un índice cardiotoráxico compatible con cardiomegalia, fracción de

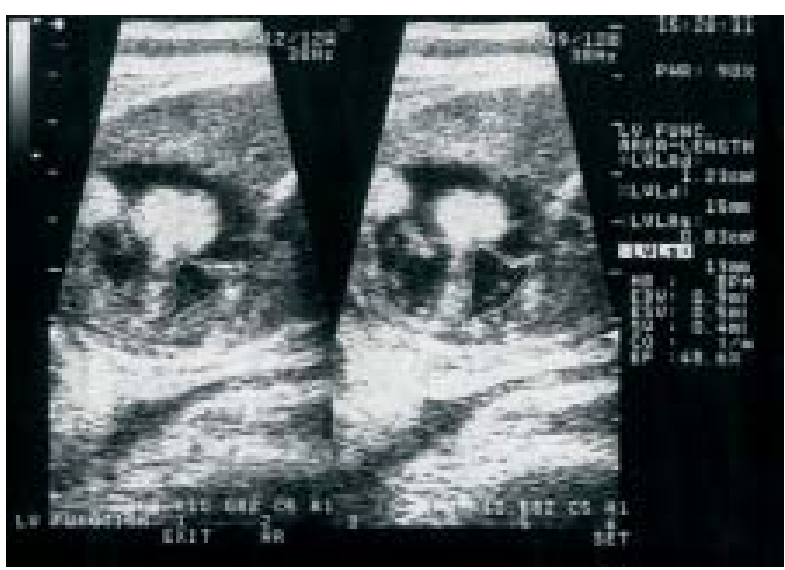

Gráfica 1. Rabdomioma en imagen de 4 cámaras.

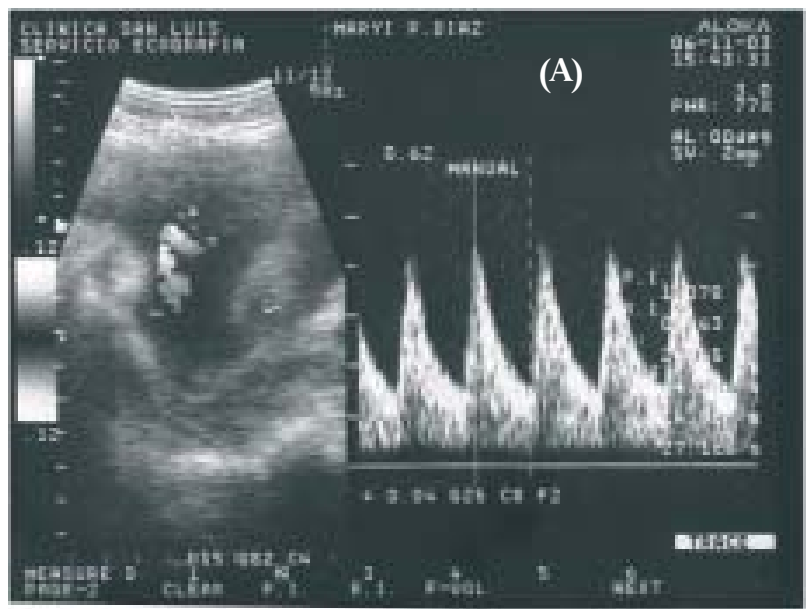

eyección del 48,6\% (FE = (EDV-ESV) /EDV x 100) del ventrículo izquierdo, y tres imágenes hiperecogénicas separadas: una en la aurícula izquierda en correlación con su tracto de salida, otra en la aurícula derecha y otra en el septum interventricular, sugestivas de rabdomioma por sus características ecográficas, asociado a un pequeño derrame pleural (gráfica 1).

El doppler fetal se informó como normal con una arteria umbilical que reporta un IR 0,66 y un ductus venoso de morfología normal con velocidad máxima de $31,6 \mathrm{~cm} / \mathrm{sec}$ (gráfica 2).

Se realizó una ecografía de detalle anatómico que reportó una ventriculomegalia de $11 \mathrm{~mm}$ (gráfica 3).

La amniocentesis reportó 51,000 cuerpos lamelares, glucosa de 43,3 mg/dl, leucocitos de $7 \mathrm{x} \mathrm{mm}^{3}$ y un gram negativo para bacterias.

El uroanálisis fue negativo para infección, la proteína $\mathrm{C}$ reactiva, negativa y el cuadro hemático, normal. Se realizó cesárea por madurez pulmonar, ruptura prematura de membranas, presentación de pelvis y antecedentes de dos cesáreas anteriores con probables signos de falla cardíaca fetal incipiente.

El producto tuvo un peso fetal estimado de 2.296 gramos, Apgar de 7 y 8, al minuto y los 5 minutos respectivamente. Se auscultó un soplo sistólico. Se solicitó una tomografía por sospecha de esclerosis tuberosa (gráfica 4), que reportó calcificaciones subependimarias.

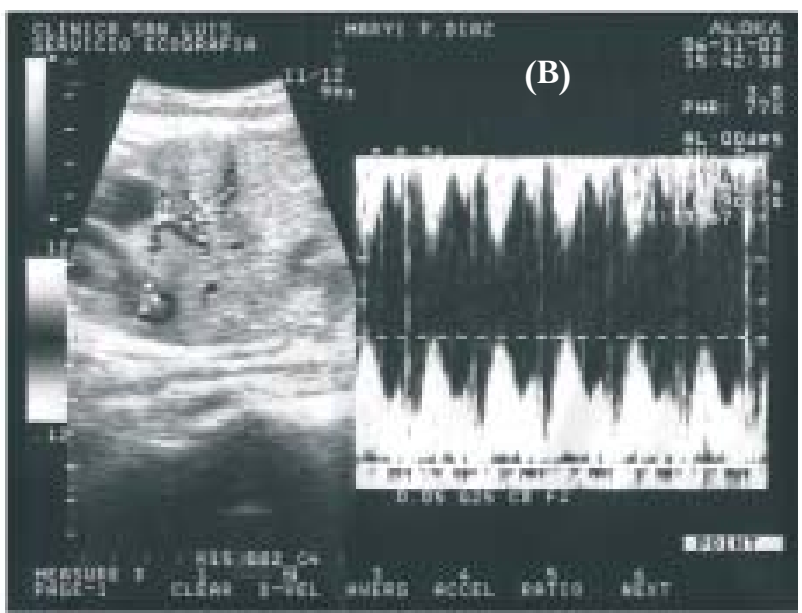

Gráfica 2. Doppler de (A) arteria umbilical y (B) ductus venoso. 


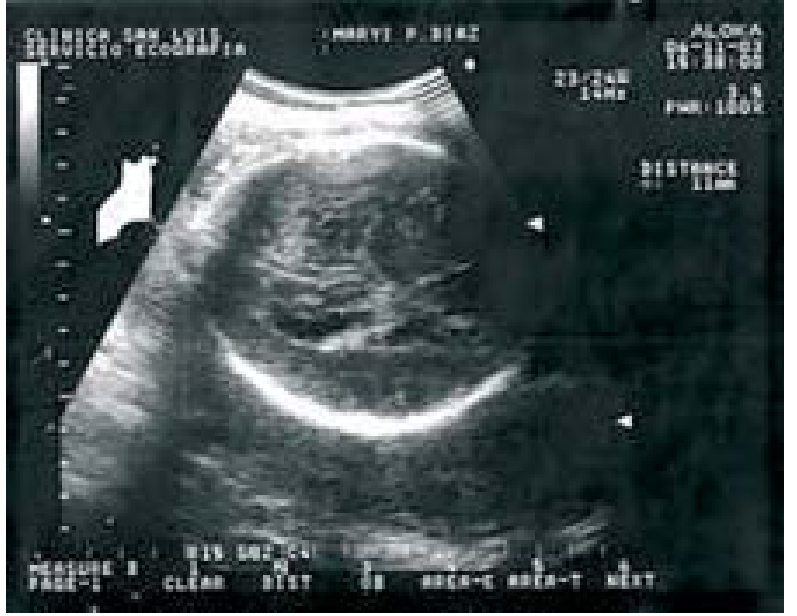

Gráfica 3. Ventriculomegalia límite.

\section{DISCUSIÓN}

La incidencia de los tumores cardíacos es muy baja. Los reportes encontrados en la literatura hacen referencia de 1 a 2 por cada 10.000 nacidos vivos; ${ }^{4}$ el 90\% de estos tumores son benignos y el $58 \%$ son rabdomiomas. Se conoce una variedad de tumores cardíacos como teratomas, mixomas, hamartomas y mesoteliomas. El rabdomioma es conocido también como hamartoma miocárdico y está formado por miocitos inmaduros; histológicamente se observa en el músculo cardíaco la presencia de células en araña con un gran contenido de glicógeno que se localizan en los ventrículos, el septum interventricular y ocasionalmente en las aurículas. ${ }^{5}$ En el caso en mención evidenció localización en el septum interventricular y aurículas, reportando una baja fracción de eyección del ventrículo izquierdo para la edad gestacional, lo que está en relación con una probable obstrucción del tracto de salida.

La esclerosis tuberosa (ET) es un síndrome neurocutáneo, autosómico dominante, que se manifiesta clásicamente por retraso mental, convulsiones, áreas de esclerosis cerebral y rabdomiomas cardíacos. ${ }^{6}$ Después de la descripción en 1992 de los nuevos criterios diagnósticos, se consideró que la presencia de rabdomiomas múltiples en un paciente es diagnóstico de ET. ${ }^{7}$

El caso en mención mostró la asociación de múltiples rabdomiomas con ventriculomegalia

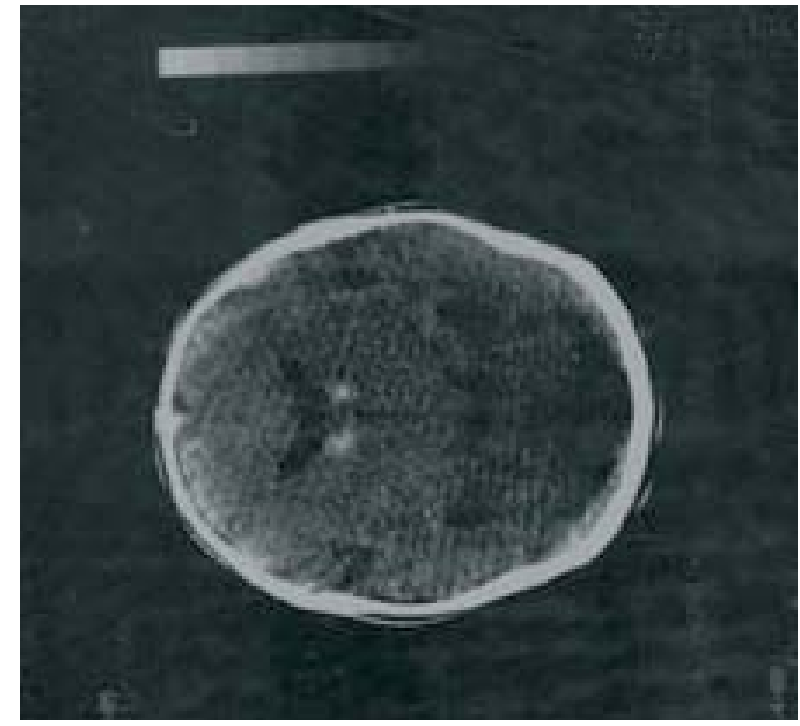

Gráfica 4. Calcificaciones en tomografía postnatal.

y calcificaciones subependimarias, sugiriendo el diagnóstico de ET. El hallazgo del rabdomioma fue casual por un ultrasonido de rutina en una ruptura prematura de membranas a las 33,6 semanas.

Ante la presencia de un rabdomioma cardíaco en un feto se sugiere el siguiente protocolo de manejo:

1. Realizar ecografía de detalle anatómico.

2. Efectuar ecocardiografía fetal.

3. Vigilar el feto, debido a la posibilidad de falla cardíaca, monitoreando la fracción de eyección y el gasto cardíaco, entre otros parámetros.

4. Realizar doppler de vena cava superior/aorta para el diagnóstico del tipo de arritmia. ${ }^{8}$

5. Iniciar medicamentos como la digoxina y/o sotalol para el tratamiento de las arritmias (gráfica 5).

6. Demostrar madurez pulmonar (amniocentesis) y considerar el parto.

7. En la etapa postnatal estaría indicado el manejo médico de las taquiarritmias y la ablación por radiofrecuencia ante la no respuesta al tratamiento farmacológico. ${ }^{9}$

8. Realizar manejo quirúrgico de la masa.

9. Iniciar tratamiento del síndrome convulsivo crónico asociado a la esclerosis tuberosa. 


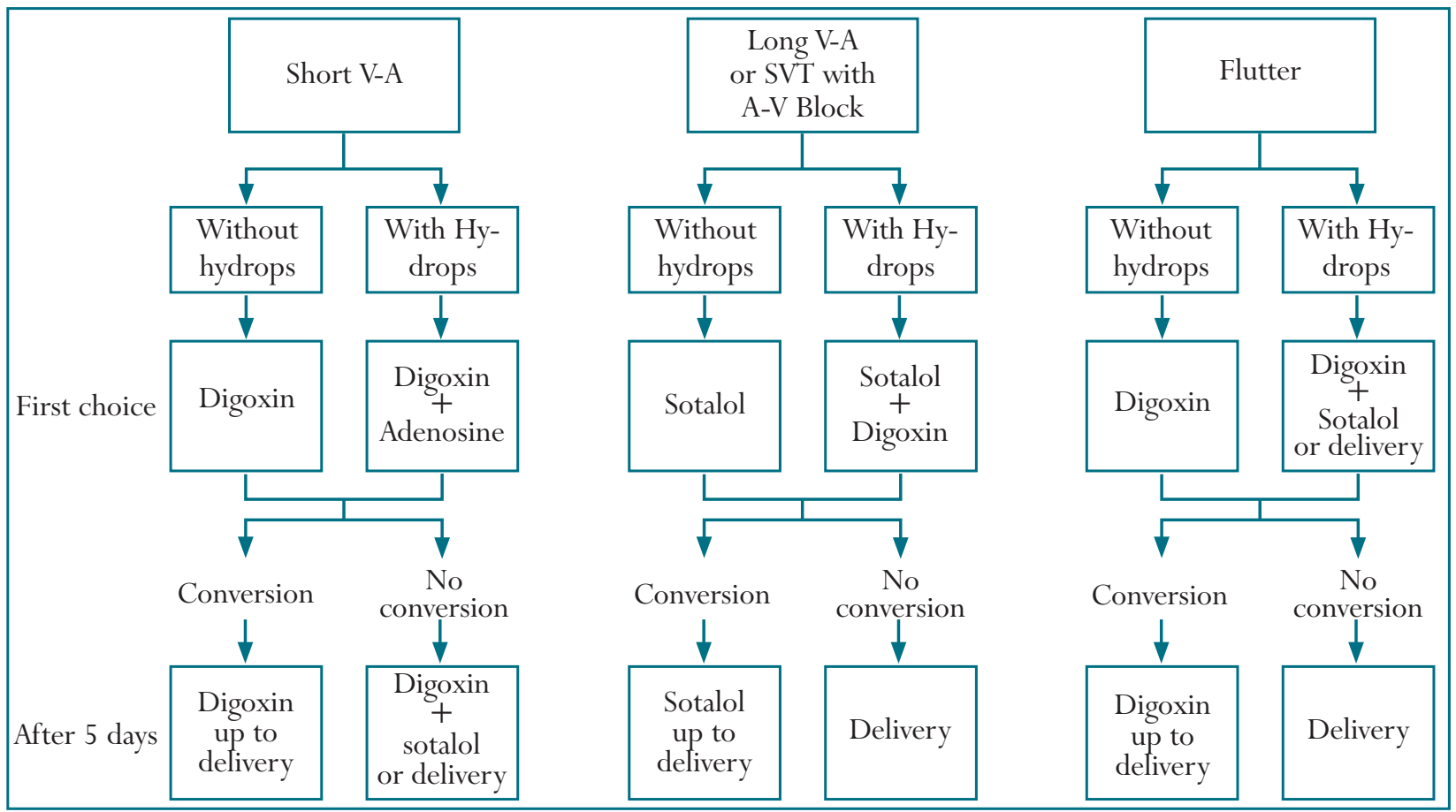

Gráfica 5. Protocolo de tratamiento para las taquiarritmias fetales. SVT, supraventricular tachycardia; VA, ventriculoatrial; AV, atrioventricular. Fuente: Fouron J-C et al. Management of fetal tachyarrhythmia based on superior vena cava/aorta Doppler flow recordings. Heart 2003;89:1211-1216. Reproducido con permiso del BJM Publishing Group.

\section{REFERENCIAS}

1. Tworetzky W, McElhinney DB, Margossian R, MoonGrady AJ, Sallee D, Goldmuntz E. Association between cardiac tumors and tuberous sclerosis in the fetus and neonate; Am J Cardiol 2003;92:487-9.

2. Bader RS, Chitayat D, Kelly E, Ryan G, Smallhorn JF, Toi A, et. al. Fetal rhabdomyoma: prenatal diagnosis, clinical outcome, and incidence of associated tuberous sclerosis complex. J Pediatr 2003;143:620-4.

3. Dalvi R, Vernekar J, Godinho S, Kartha R. Cardiac rhabdomyoma - antenatal diagnosis. Ind J Radiol Imag 2002;12:339-40.

4. Mcallister H Jr. Primary tumors of the heart and pericardium. Pathol Annu 1979;14:325-30.

5. Bosi G, Linterman JP, Pellegrino PA, Svaluto-Moreolo G, Vliers A. The natural history of cardiac rhabdomyoma with and without tuberous sclerosis. Acta Pediatr 1996;85:928-31.
6. Muhler EG, Turniski-Harder V, Engelhardt W, Von Bermuth G. Cardiac involvement in tuberous sclerosis. Br Heart J 1994;72:584-90.

7. Roach ES, Smith M, Huttenlocher P, Bhat M, Alcorn D, Hawley L. Diagnostic criteria: tuberous sclerosis complex. Report of the diagnostic criteria committee of the national tuberous sclerosis association. J Child Neurol 1992;7:221-4.

8. Van Hare GF, Witherell CL, Lesh MD. Follow-up of radiofrequency catheter ablation in children: results in 100 consecutive patients. J Am Coll Cardiol 1994;23: 1651-9.

9. Jaeggi E, Fouron JC, Fournier A, Van Doesburg N, Drblik SP, Proulx F. Ventriculo-atrial time interval measured on M mode echocardiography: a determining element in diagnosis, treatment, and prognosis of fetal supraventricular tachycardia. Heart 1998;79:582-7. 